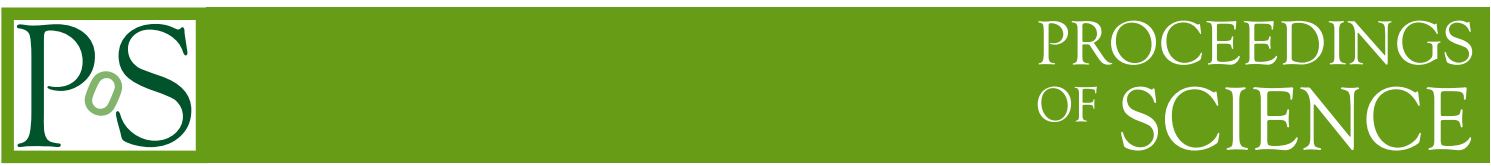

\title{
Performance of the ARIANNA Hexagonal Radio Array
}

\author{
Corey Reed* for the ARIANNA Collaboration \\ Department of Physics and Astronomy, University of California, Irvine \\ Irvine, CA 92697-4575, USA \\ E-mail: cjreedeuci.edu
}

\begin{abstract}
Installation of the ARIANNA Hexagonal Radio Array (HRA) on the Ross Ice Shelf of Antarctica has been completed. This detector serves as a pilot program to the ARIANNA neutrino telescope, which aims to measure the diffuse flux of very high energy neutrinos by observing the radio pulse generated by neutrino-induced charged particle showers in the ice. All HRA stations ran reliably and took data during the entire 2014-2015 austral summer season. A new radio signal direction reconstruction procedure is described, and is observed to have a resolution better than a degree. The reconstruction is used in a preliminary search for potential neutrino candidate events in the data from one of the newly installed detector stations. Three cuts are used to separate radio backgrounds from neutrino signals. The cuts are found to filter out all data recorded by the station during the season while preserving $85.4 \%$ of simulated neutrino events that trigger the station. This efficiency is similar to that found in analyses of previous HRA data taking seasons.
\end{abstract}

The 34th International Cosmic Ray Conference,

30 July- 6 August, 2015

The Hague, The Netherlands

${ }^{*}$ Speaker. 


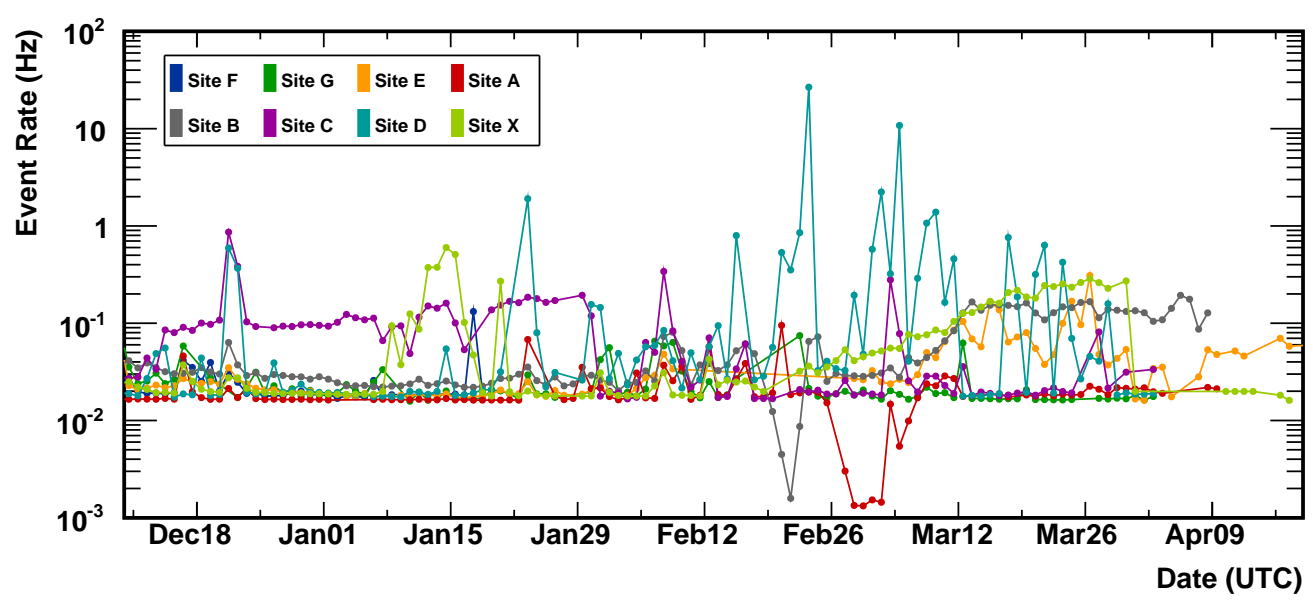

Figure 1: Daily average trigger rates of each HRA station during the 2014-2015 data taking season. See text for details.

\section{The ARIANNA Hexagonal Radio Array}

The ARIANNA Collaboration plans to construct a neutrino telescope capable of measuring the diffuse flux of high energy neutrinos in the $10^{8}-10^{10} \mathrm{GeV}$ range [1]. The ARIANNA Hexagonal Radio Array (HRA) serves as a pilot program to the full ARIANNA telescope. During the 20142015 austral summer, the HRA installation was completed at the ARIANNA detector site on the Ross Ice Shelf of Antarctica [2]. It consists of eight independent detector stations, each of which has four Log-Periodic Dipole Antenna (LPDA), an autonomous data acquisition (DAQ) system (with local data storage and remote data transfer capabilities) as well as local solar power. The stations measure radio pulses in the $50 \mathrm{MHz}-1 \mathrm{GHz}$ frequency range, making them sensitive to the radio emission produced by charged particle showers generated by neutrino interactions in the ice, known as the Askaryan effect $[3,4,5]$. Details on the station hardware may be found in Refs. [2, 6].

All eight stations were installed by early December, 2014 and ran until the sun began to set around early April, 2015. The layout of the detector stations can be found in Refs. [1,2]. Figure 1 shows the trigger rate of each station during the 2014-2015 austral summer data taking season.

Similar to previous seasons, trigger rates were generally low and minimal threshold tuning was required. While each station is similar, small variations can lead to noticeable differences in the trigger rates. The majority of Site F data has not yet been transferred off station and does not appear in the figure. During late February and early March, the trigger requirements at Sites A and B were temporarily increased to lower the rates. This was done to test the reliability of sending radio waveform data off Antarctica using satellite communications [2] limited to 340 bytes per message. The strong solar burst on December 20, 2014 is visible in a correlated rise of trigger rates on all stations [2] (it is also visible in Fig. 6, discussed in Sect. 3.3). The higher rates of Site C early in the season are simply due to low triggering thresholds (which were later corrected). The high rate spikes of Site D later in the season are caused by radio bursts emitted when the station's battery switches on (station powered by battery) and off (station powered directly by solar panels). 
This will be corrected during the 2015-2016 deployment season by installing a new battery inside the radio-tight DAQ box. Other rate increases are correlated with storms (i.e. February 8) and the dramatic temperature drop that occurs during the beginning of March. As the temperature falls from about $0^{\circ} \mathrm{C}$ to $-20^{\circ} \mathrm{C}$, the gain of the amplifiers increases, leading to a rise in trigger rates unless thresholds are adjusted to compensate.

\section{Radio Signal Direction Determination}

The direction from which radio signals arrive at a detector station is reconstructed using the cross-correlation of the time dependent radio signals observed by parallel LPDAs. Each HRA station has four LPDAs, arranged symmetrically around the station DAQ box. Antennas situated across from each other (not adjacent) are oriented with parallel tines and separated by $6 \mathrm{~m}$. Thus there are two such parallel LPDA pairs, so that a signal perfectly co-polarized with one antenna pair will be perfectly cross-polarized with the other antenna pair. Cross-correlations are used to determine the likelihood that the measured data is consistent with the expected time delays between parallel channels for a radio signal (with a planar wave-front) arriving from a particular direction.

The most likely radio arrival direction is determined by comparing two separate fit procedures. The first procedure uses a genetic minimization algorithm to choose the most likely arrival direction. The second procedure simply scans the entire angular phase space in $1^{\circ} \times 1^{\circ}$ bins. In both procedures, the arrival direction estimate is further refined using the Migrad minimization algorithm. The two resulting fits are compared and the one with the best likelihood is chosen.

Special calibration data has been collected in order to quantify the radio pulse arrival direction resolution. A Pockels Cell Driver (PCD) is used to generate a very short (about 3 ns) uni-polar pulse that is transmitted from an LPDA. The transmitting antenna is oriented with its boresight vertically downward and placed at various positions on the ice around a station. The calibration pulse travels downward through the firn and ice, reflects off the sea water below the ice shelf and then travels up through the ice and firn where it is measured by the station. The reflection has been observed to preserve polarization [7]. The station is triggered externally in order to veto radio noise produced by the PCD pulser.

Figure 2 shows the reconstructed transmitter location, relative to the station located at the origin, for events in three sets of calibration data. The true transmitter positions are shown by the blue markers. At each location, the transmitting LPDA is oriented such that its tines are at a $45^{\circ}$ angle to the tines of all four receiving LPDAs.

The angular deviation between the reconstructed signal direction and the true direction is shown in Fig. 3. This represents the radio pulse direction resolution, not a neutrino direction resolution. The neutrino direction resolution is expected to be on the order of a few degrees, mainly due to the finite resolution with which the polarization of the radio signal can be determined [1].

\section{Search for Neutrinos in Site B Data}

The station at Site B has a configuration that, among currently deployed HRA stations, contains the most pieces of hardware planned for use in a full ARIANNA station. This includes a new signal digitizing chip, amplifiers with a flatter response over the frequency bandwidth and a 


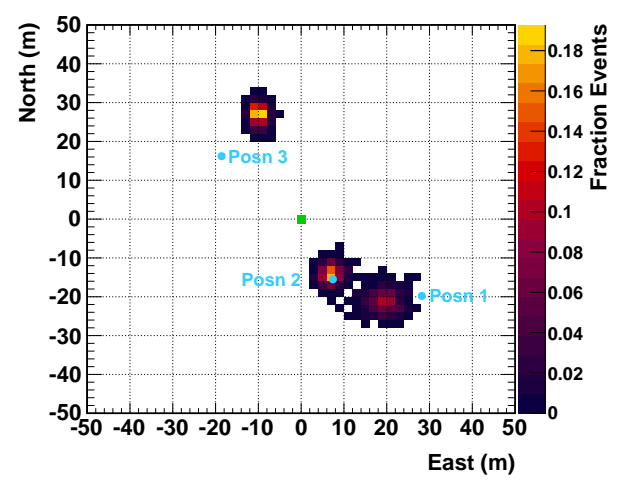

Figure 2: The reconstructed calibration pulse transmitter location. The true transmitter locations are shown by the blue markers. The station sits at the origin, marked by the green square. The transmitter LPDA is always oriented such that the plane of its tines are offset by 45 degrees from those of each receiving LPDA.

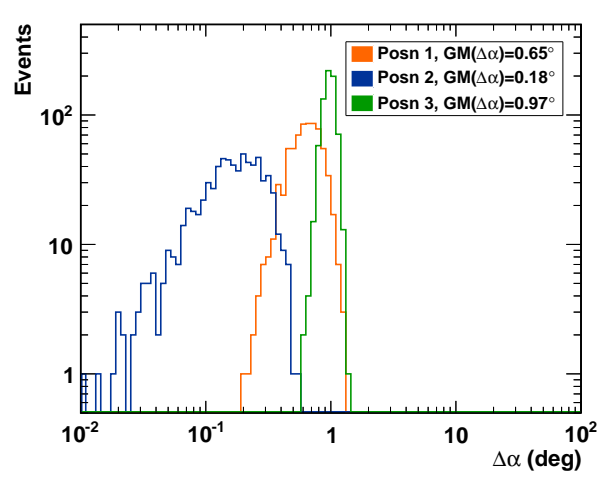

Figure 3: The angular deviation of the reconstructed radio pulse arrival direction from the expected direction for events shown in Fig. 2. The resolution is quantified by the geometric mean of each distribution, shown in the legend. Note that this does not represent a neutrino direction resolution.

reliable battery. A preliminary search for neutrino signals in the data taken by this station has been conducted (although no such signals are expected given the small aperture and short exposure time of this single station).

Data taken by Site B from December 10, 2014 (UTC) until the end of the season, around April 9, 2015 (UTC), is used in the analysis described in this proceeding. Data collected by the station but not yet transferred off Antarctica, most of which was taken during the last weeks of March, is not yet included in the analysis. Also excluded from the analysis is calibration data and data collected while the station's communication peripherals were powered on. This latter data is not particularly noisy and may be included in future analyses, but has been excluded here for simplicity. The analyzed data set comprises 93.1 days of livetime, corresponding to about a $90 \%$ uptime [2] during the data taking periods used in the analysis. The $10 \%$ downtime is due to the frequency with which the station transmits its data off Antarctica. This is tuneable remotely and my be reduced in the future, but was deemed an acceptable cost given the benefits of receiving station data in near real time.

A set of neutrino simulation events have been generated according to the procedure described in Ref. [1]. The same trigger is applied in the generation of the neutrino signal simulation as has been applied in the Site B data set. Namely, that two of the four receiving LPDAs show a bipolar pulse having both the high and low crests extending beyond the high and low thresholds, each set at four times the thermal noise RMS.

\subsection{Rejecting Single Frequency Resonance Events}

Some events collected by the station contain a large amount of their power at a single frequency. Such waveforms stand in stark contrast to the waveform expected for a neutrino, which contains significant power across the $50 \mathrm{MHz}$ to $1 \mathrm{GHz}$ frequency band due to the very short time duration of the Askaryan pulse. These "sinusoidal" events in the data are likely caused by detector 

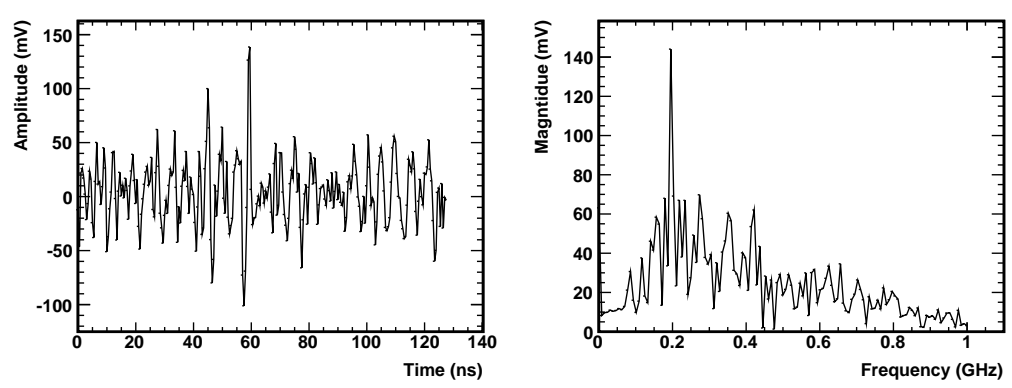

Figure 4: Example of a waveform displaying a strong resonance near $200 \mathrm{MHz}$ that is filtered out by the $\eta$ cut. This waveform is from the north LPDA. The event was recorded at Site B on March 13, 2015 at 16:46:19 UTC. Left: the waveform in the time domain. Right: the waveform in the frequency domain.

electronics, such as radio noise emitted by the external charge controller of the battery switching on and off. An example of such an event is shown in Fig. 4.

Potential neutrino candidate events are required to have a significant amount of power at several different frequencies. This is done by first calculating the discrete Fourier transform of the voltage versus time measured by an LPDA (after amplification). The frequency bin containing the largest amount of power is then identified; $p_{\max }$ represents the amount of power in this bin. The number of frequency bins that contain more than $p_{\max } / 4$ on the LPDA is called $\eta_{L P D A}$. The smallest $\eta_{L P D A}$ value in the event is defined as $\eta$ for the event.

Potential neutrino candidates are required to have $\eta>3$ frequency bins. After applying this cut, $74.3 \%$ of the Site B data remains, while $99.3 \%$ of simulated neutrino events that trigger the station survive.

\subsection{Finding External Pulses}

The vast majority of triggers recorded by the station are caused by continuous thermal radio emission. These events are distinguished from neutrino pulses as they show little to no correlation between parallel LPDA measurements.

The angular reconstruction procedure described in Sect. 2 is used to identify events that may have been produced by an external radio pulse. The angular direction fit likelihood, $-\log (L)$ is required to be reasonably good for potential neutrino candidate events. The cut on the likelihood is fairly loose, $-\log (L)<1.7$, for low amplitude events and becomes stronger, $-\log (L)<0.5$, for high amplitude events.

The fit likelihood cut is shown as a function of the peak to peak amplitude in Fig. 5 for neutrino simulations (left), Site B triggered data (middle) and pure thermal noise data recorded at Site B using software forced triggers (right). The peak to peak amplitude of an event is quantified by calculating the peak to peak value of the waveforms on all LPDA channels of an event, and keeping the smallest peak to peak value. Events outside (below) the blue shaded region are kept as potential neutrino candidates.

This cut removes nearly all of the purely thermal events recorded by Site B. Only $2.7 \%$ of the Site B data events have both $\eta>3$ and $-\log (L)<f(p)$, where $f(p)$ is a function of the minimum peak to peak amplitude of the event. The function $f(p)$ is shown by the lower edge of 

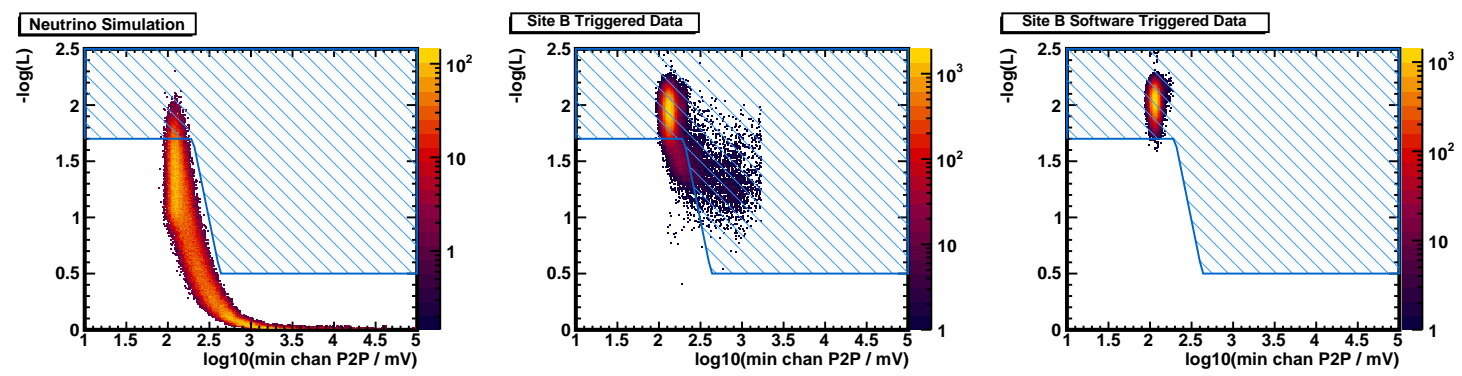

Figure 5: The fit likelihood versus the log of the peak to peak signal size on the LPDA channel with the smallest peak to peak signal in three different data sets. Left: the neutrino simulation. Middle: Site B triggered data. Right: Site B software triggered data (pure thermal noise). Events in the blue shaded region do not pass the fit likelihood cut. Only events passing the $\eta>3$ cut are shown for each data set.

the blue region in Fig. 5. Of the simulated neutrino events that trigger the station, 96.0\% survive the application of both cuts.

\subsection{Finding Neutrino-Like Signals}

After the application of the $\eta$ and $-\log (L)$ cuts, many of the events remaining in the Site B data set are non-thermal radio pulses. Much of this data was recorded during periods of high winds as weather systems pass over the ARIANNA site on the Ross Ice Shelf. During the 20142015 data taking season, the largest storm occurred during February. From February 6-8, winds above 40 knots were recorded at New Zealand's Scott Base, about $100 \mathrm{~km}$ from the ARIANNA site and over Minna Bluff. Wind data is not currently available for the ARIANNA site itself. A second important source of the non-thermal Site B data is the battery electronics, which emit radio frequency noise when switching on and off. This occurs toward the end of the season, as the station alternates drawing power from the battery and solar panels while periods of strong sunlight become shorter and less frequent.

Simulations of the time dependent waveforms produced by neutrino signals are used to distinguish background radio pulses from potential neutrino observations. The time dependent neutrino simulations are described in Refs. [1, 8]. The simulations include the measured responses of the ARIANNA LPDA as well as the amplifiers used at Site B, which play a large role in determining the expected waveform recorded upon observation of a neutrino-induced Askaryan pulse.

For a given event, the reconstructed signal direction is used to determine the arrival direction of the signal in the local frame of the LPDA. The neutrino waveform expected for a signal probing this part of the antenna response (and passing through the amplifiers of Site B) is then looked up from a table of such neutrino waveform templates. The template lookup table is binned in $10^{\circ} \times 10^{\circ}$ wide bins in the local frame of the antenna. Thus, the reconstruction presented in Sect. 2 is much more accurate than necessary for the analysis presented in this proceeding.

The cross-correlation between the chosen neutrino waveform template and the actual recorded waveform is then calculated. The maximal value of this correlation (at any time offset) is defined as $\xi_{L P D A}$. Because the absolute polarization of the potential neutrino signal is not determined in the 


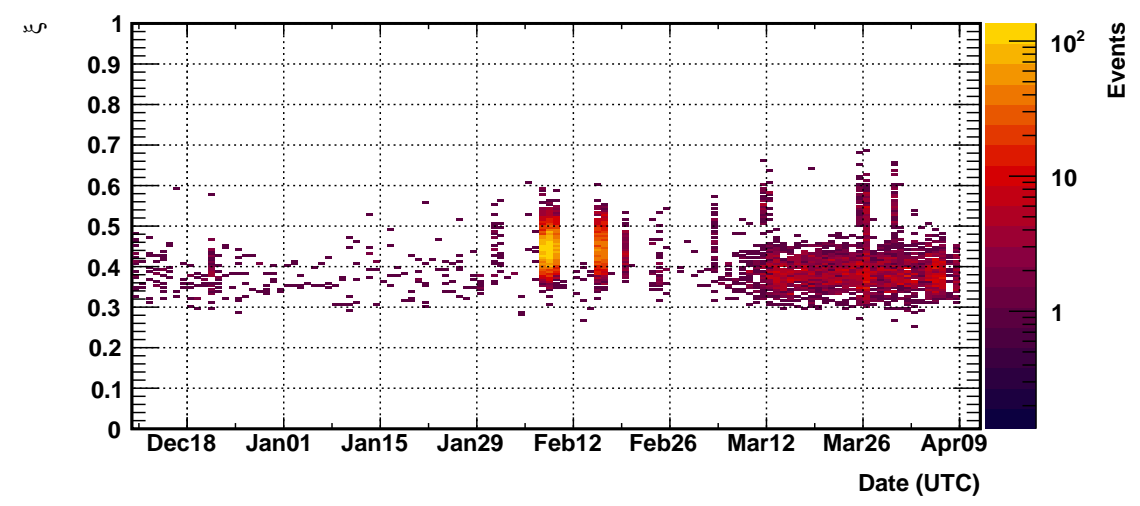

Figure 6: The daily $\xi$ distribution of events recorded by Site B that pass both the $\eta<3$ and $-\log (L)<f(p)$ cuts. The solar burst is visible in mid December. The large storm in early February is also visible. The increased number of events with poor $\xi$ values toward the end of the season is a result of the higher trigger rates, due to a combination of colder temperatures and radio noise emitted by the battery turning on and off.

data, it is not known whether the neutrino template or its inverse should compare more favorably to the recorded waveform. Therefore, both are tried and the larger value is assigned to $\xi_{L P D A}$.

A new cut variable, $\xi$, is then defined as the largest $\xi_{L P D A}$ observed on any LPDA channel. Figure 6 shows the distribution of the $\xi$ variable in 24-hour wide bins over the course of the season for events recorded at Site B that pass both the $\eta<3$ and $-\log (L)<f(p)$ cuts.

Potential neutrino candidate events are required to have $\xi>0.7$. None of the Site B data survives the application of all three cuts, while $85.4 \%$ of the simulated neutrino events that trigger the station survive the $\eta>3,-\log (L)<f(p)$ and $\xi>0.7$ cuts. This signal efficiency is comparable to that seen in the analyses of previous HRA season data sets.

The preliminary analysis of the data collected at Site B between December 10, 2014 and April 9, 2015 has produced a set of simple selection criteria that are able to separate potential neutrino candidate events from radio backgrounds in the data. Data taken from the other HRA stations is currently under analysis. A search for neutrino signals in the combined 2014-2015 season HRA data is expected to improve upon the diffuse neutrino flux limit presented in Ref. [1], assuming no neutrino candidates are found in the data.

\section{Conclusions}

The complete HRA detector has been installed at the ARIANNA site on the Ross Ice Shelf of Antarctica. All eight stations ran reliably and took data from the time the installation crew departed the site in early December until the sunlight faded in late March to early April. The installation performed during the 2014-2015 austral summer made use of upgraded detector hardware, some of which is described in Ref. [2].

A radio signal direction reconstruction package has been developed and applied to both calibration pulse data as well as to triggered data recorded by the stations. The angular resolution of the reconstruction is found to be on the order of or better than a degree in both simulations and calibration data. 
The reconstruction has been used in a preliminary search for neutrino signals in the 93.1 days of data recorded by the station situated at Site B. Three cuts are employed by the potential neutrino candidate search to find events that (a) have power at multiple frequencies, (b) fit well to an incoming plane wave and (c) have waveforms resembling those expected for a neutrino.

The vast majority of data removed by these cuts are due to simple thermal radio noise. Similar to previous seasons, the majority of non-thermal backgrounds are recorded during periods of high winds at the ARIANNA site. This season, some noise has been observed originating from the station battery while it is switched on and off by its charge controller. In future seasons, batteries will be deployed inside the radio-tight DAQ boxes to eliminate this radio noise source.

No events recorded by the station at Site B pass the application of all cuts, while $85.4 \%$ of simulated neutrino events that trigger the station survive the cuts. This signal efficiency is comparable to that obtained in analyses of previous HRA data taking seasons [1]. A similar analysis of the data collected by all HRA stations is currently underway, after which a limit on the diffuse neutrino flux will be placed (assuming no neutrino candidates are found in the data).

The authors thank the staff of Antarctic Support Contractors, Lockheed, and the entire crew at McMurdo Station for excellent logistical support. This work was supported by funding from the Office of Polar Programs and Physics Division of the US National Science Foundations, grant awards ANT-08339133, NSF-0970175, and NSF-1126672. A. Nelles is supported by a research fellowship of the German Research Foundation (DFG), grant NE 2031/1-1.

\section{References}

[1] ARIANNA Collaboration, S. Barwick et al., A First Search for Cosmogenic Neutrinos with the ARIANNA Hexagonal Radio Array, Astropart.Phys. 70 (2015) 12-26, [arXiv: 1410 . 7352 ].

[2] A. Nelles and C. Persichelli for the ARIANNA Collaboration, Measuring Neutrinos with the ARIANNA Hexagonal Radio Array, These Proceedings (2015).

[3] G. A. Askaryan JETP 14 (1962) 441.

[4] G. A. Askaryan JETP 21 (1965) 658.

[5] ANITA Collaboration, P. Gorham et al., Observations of the Askaryan effect in ice, Phys.Rev.Lett. 99 (2007) 171101, [hep-ex/0611008].

[6] ARIANNA Collaboration, S. W. Barwick et al., Design and Performance of the ARIANNA HRA-3 Neutrino Detector Systems, IEEE Trans.Nucl.Sci., (in press) (2014) [arXiv: 1410 . 7369].

[7] ARIANNA Collaboration, S. W. Barwick et al., Radio-frequency Attenuation Length, Basal Reflectivity, Depth, and Polarization Measurements from Moore's Bay in the Ross Ice-Shelf, J.Glaciol. 61 (2015), no. 227 [arXiv: 1410 . 7134].

[8] ARIANNA Collaboration, S. Barwick et al., Time Domain Response of the ARIANNA Detector, Astropart.Phys. 62 (2014) 139-151, [arXiv:1406.0820]. 